\title{
Editorial The Importance of Developing Narrative Capacity
}

Jennifer Lehmann

Welcome to our first Issue of Children Australia for 2018. We hope you have had a wonderful Christmas and entered the New Year with energy and enthusiasm for the challenges ahead. We also welcome back many of our Editorial Consultants and especially want to make our new members of the team feel engaged in the journal's activities for 2018. One of our new Editorial Consultants is Shraddha Kapoor who is Associate Professor at Department of Human Development and Childhood Studies, Lady Irwin College, University of Delhi. Dr Neerja Sharma, now retired, who has supported Children Australia for some years, was Shraddha's Professor before becoming her colleague and now a dear friend. Shraddha herself has been teaching in the department for last 27 years in the subjects of developmental psychology, child development, wellbeing, family and gender. Her particular interests are childcare, early childhood education and gender.

A second new member of the group is Nicola Atwool who is Associate Professor in the social work programme at the University of Otago. Prior to her return to the university at the beginning of 2012, she spent 6 years as a principal advisor in the Office of the Children's Commissioner. She had previously worked at the University of Otago as a lecturer and senior lecturer in the social work programme from 1994 to 2005, and has also maintained a close relationship with the Children's Issues Centre during her time at the institution. Nicola has professional qualifications in social work and child and adolescent psychotherapy, and was employed in a variety of roles by the statutory care and protection and youth justice service for nearly 20 years before taking up an academic position. Her research interests include the social construction of childhood and adolescence, attachment theory, resilience, the impact of trauma on children and young people, the experience of children in care and social work intervention with children, young people and their families. Nicola has completed two major projects involving children in care: a PhD in 2008 exploring the impact of attachment assessments on decision making for children in care, and a report on the quality of services provided for children in care in 2010 while working at the Office of the Children's Commissioner. More recently, her publications have focused on transition from care, birth family contact for children in care and Life Story work.

And we have pleasure in welcoming another colleague hailing from New Zealand, Dr Michael Gaffney. Michael is a lecturer in early childhood education at the University of Otago, College of Education in Dunedin. Before this he worked at the University of Otago's Children's Issues Centre, which is an interdisciplinary research and teaching centre. This provided Michael with the opportunity to conduct work in disability, childhood studies, early childhood education and schooling policy, and social and educational evaluation.

In the course of 2018, we hope to bring you two special issues, one in June and the other in September. The June issue will be led by Dominic McSherry, a Senior Research Fellow with the Centre for Evidence and Social Innovation (CESI) in the School of Social Sciences, Education, and Social Work (SSESW) of Queen's University, Belfast. The September issue will be led by Jonathon Sergeant who is a Senior Lecturer with the Faculty of Education and Arts at Australian Catholic University. Their assistance and initiative in developing these two issues is much appreciated.

In developing editorial comments for this March Issue, it occurred to me that it is a while since the nature of narrative in children's lives has been a topic in this journal. It is certainly not one that can be easily dealt with in a page or two, but I have been interested to see how some of the current studies are now linking the issues of narrative development with aspects of creativity, development of identity and capacity to cope with trauma. At a time when neuroscience and brain development underpins our ideas about the impacts of neglect and trauma, it is timely to consider the role that narrative (defined in the broadest sense) plays in children's development.

Many people think of narrative as spoken language, words and literacy. However, teaching children to read and write is but one small part of developing our capacity for narrative, which really starts at birth - and perhaps even prior to this - as we hear, feel and interact with others. Recently, respected music educator Gill (2017, p. 25) 
pointedly observed that 'Many educators believe that literary and numeracy are actual subjects or disciplines' when they are instead 'states or conditions at which one arrives as a result of being educated'. Far more important are the foundations for literacy and numeracy, 'the things which enrich our existence: our music, our literature, our painting, our sculpture, our decorative art', and Gill stresses 'our obligation to constantly nurture and enrich creativity through rigorous, imaginative and serious education programs in our schools'. Gill's emphasis on the inclusion of many expressive forms, and the capacity of creative activity to promote the ongoing development of literary and numeracy throughout our lives, is closely allied, I think, to the importance of our capacity to develop narratives about ourselves and the world around us. Our sense of ourselves is storied, our perspective developed through our experiences, each of us a little different as we work constantly to form and reform our identity in the light of events, relationships and challenges. Words are important, of course, as words and sounds give expression to our thoughts and feelings, and the manner in which they are put together can be extremely powerful.

Talking to children and, through our interactions with them, helping them create connections with the physical and social environment, provides children with a foundation for language that can become richer and more nuanced over time. Children swiftly learn the subtleties of tone and emphasis, the use of silence and, later, the role of omission of information, the casting of character, manipulation of logic and all those other complexities of communicating. But first we need to engage in exchanges, verbal and otherwise, that create context and coherence. Seemingly simple stories for toddlers are actually profound in helping children to follow narrative structure, but also provide the building blocks for narratives they create about themselves. Stories - narrative accounts - can be very brief and yet meaningful. Take, for instance, a mother's account of her child's crying: 'Poor bubs, you've wet your nappy. Let's see what we can do to get you cleaned up'. Seventeen words in this narrative are all it takes to convey that the mother:

- empathises with the distress of her child - I'm concerned about you and for your sense of wellbeing and I am emotionally engaged with you

- can provide a logical explanation - a reason for feelings of distress

- legitimates the distress rather than ignoring or dismissing it

- offers a solution - doing something active together to gain resolution

- engages in the action and demonstrates responsibility

- has the capacity and the agency to bring about change and comfort

- demonstrates calmness in the face of emotional display, assuring the child that being wet is not alarming.
Imagine, then, the truly amazing array of messages embedded in narratives over the course of even a single day.

Narrative, as a tool and as a process to promote learning, includes talking about others too, an example being: 'Dad will be home soon. He'll pick up your brother on the way and then we'll have tea'. A mere 18 words, maybe, but more than enough to allow a child to learn about:

- people being able to leave and return

- a parent having agency in reuniting with a family member on the way to re-joining those at home - an additional step in a journey

- being able to carry out travel safely

- siblings being different to one another in terms of age, activity, location outside the home

- family rituals - parents planning for reunion and a meal

- a family culture of respect, indicated by waiting for others and being patient.

As children grow older they discover narratives within silence and omissions too. Silences may express many things including, more obviously, sadness and/or anger. And children observe the silence stories between parents even if they don't yet have the language capacity to provide an explanation for what they are experiencing.

In the last few decades there has been an enormous amount of research about the emotional development of children, and it has been demonstrated that children's narrative coherence is a predictor of emotional competence. A recent study carried out by Berzenski and Yates (2017) focused on children aged 4, 6 and 8 years, and found that:

Importantly, supportive parenting in the context of children's encounters with challenging tasks entails not only parental positive regard and expressions of praise, but also the capacity to acknowledge and contain the child's negative affect and frustration. Therefore, in addition to providing examples of positive emotional displays, supportive presence by parents early in development may include negative emotional displays that scaffold the child's capacity to engage in their own positive and negative emotional displays. In this supportive context, children can learn to acknowledge and label emotions rather than become overwhelmed and disorganized by them. Exposure to parents' responsive and congruent emotional displays may be particularly important during the preschool years when children begin to become proficient in recognizing discrete emotions. During this period, it may be that the presence of the parent's contextually appropriate emotional displays and support for the child's expression of the full range of emotions begin to set the stage for emotion recognition (p. 1919).

This work assumes a capacity to provide narrative, which Stagnitti, Bailey, Stevenson, Reynolds, and Kidd (2016) found is strongly associated with active use of language and play. Research 'on play and language in preschool and school-age children has linked play to developments in narrative skills such as story comprehension and story 
production' (Stagnitti et al., 2016, p. 391), which are necessarily the precursors for children's narrative abilities and coherence. In addition, Stagnitti and Lewis (2015, p. 148) found 'the quality of pretend play in 4-5 year olds is important for semantic organization and narrative re-telling abilities in the school-aged child'.

The research findings that address language and social skills become particularly relevant when children have been exposed to disadvantage, abuse or neglect. There is a substantial body of literature that links language and social behaviours to contemporary concerns about how best to overcome the potential deficits in language usage, especially for those children and young people who lack proficiency and are unable to provide narrative accounts in critical circumstances, for instance when questioned by teachers, police or social workers. Whilst the results of studies vary to some extent and, at an individual level, there are children who are able to meet the standard age-related developmental expectations in spite of disadvantage, some patterns have emerged. Lum, Powell, and Snow (2018, p. 71) are conducting contemporary research in this area and state that 'Statistically significant associations were found between children's language skills and female as well as male carers' education level. The direction of the correlation indicates that higher carer education levels were associated with higher language scores in the children'. This suggests that we need to be vigilant about extending the language and thus narrative capacity of those in out-of-home care (OOHC), but also include attention to what carers are able to contribute in this area of development, as well as potential activities and services addressing the development of language skills that can be offered by human service professionals.

The acquisition of language and narrative capacity is essential for the development of a coherent 'life story' and what is called 'autobiographic reasoning'. As outlined by Reese et al. (2017, p. 613), '[d] etailed and resolved narratives of critical events in one's life, including turning-point events, are believed to be vital for one's narrative identity' and 'the connection between life narratives and well-being for adults has now been replicated many times' (p. 615). Again, for children and young people experiencing disadvantage, there are risks to their wellbeing if language and narrative capacities are not developing. One of the challenges arising when children have experienced trauma is what Valentino et al. (2014) refer to as overgeneral memory (OGM), and this may be a predictor of future mental health issues. The Valentino et al. (2014) study hypothesised 'that individual differences in maternal reminiscing style may be important predictors of OGM, or its inverse, autobiographical memory specificity, among children' (p. 1198). On completion of their analysis, the authors reported that:

it is not the extent to which details of memories are repeated, rehearsed, or elaborated that are related to children's ability to retrieve specific autobiographical memories. Rather, assisting children in making sense out of their past emotional experiences in a sensitive, supportive, and coherent way appears to be most relevant to children's memory specificity. By being sensitive to and tolerant of children's emotions as well as by helping children to create a coherent narrative about past events and to resolve negative emotions, reminiscing characterized by emotional support and coherence, in particular, may help children to understand past memories in ways that increase connections with other past events and aspects of the self (p. 1204).

The findings of these studies, and others that demonstrate the significance of language and narrative development, should come as no surprise to those working with children and young people who have experienced trauma, neglect and disadvantaged circumstances. The core issue is how to assist children, who do not necessarily have predictable and reliable access to parental support and nurturing, to develop the narrative structures and capacities that will see them avoid further deficits in development. There have been a number of approaches used over the years, some of these quite formal and requiring expertise, such as narrative therapeutic interventions (see for instance the Tree of Life Program http://www.relationshipsvictoria. com.au/training/training/course/131/11 and Richard Rose's work at https://www.childhoodinstitute.org.au/Richard Rose), while others are less formal and have relied on carers to play a key role. While much of the research refers to maternal or parental input, there is no doubt that we are concerned here about the primary and significant parenting figure in a child's life to fulfil this particular developmental need. It is in the co-construction of narrative that the child is provided with the scaffolding required and this is, in essence, conversation (Kelley \& Bailey, 2012). The engagement and exchanges between child and adult are used to build a coherent understanding of events and experiences, a sense of self and participation in relationships, capacity to identify emotions and gradually to manage these in a mature fashion.

However, for children and young people in foster care, the nature of the foster family culture and values may limit their development. In relation to education, McHugh and Pell (2013, p. 23) report that:

Research into the impact of foster parent expectations and behaviour on the educational achievement of children and young people in foster care shows that:

- Those foster children doing most poorly educationally were likely to be with foster parents who had low expectations for their achievement, had less contact with the child's school and were less likely to help with homework; and

- When there is a 'learning culture' in the foster home, the care environment can positively influence the foster child/children's educational experience. For instance, a UK study showed that improving the home learning environment - by reading to children, providing books 
and increasing computer access - had positive educational benefits for foster children.

One could anticipate that engaging in conversations for life learning would also be limited if learning and education are not highly valued. In a small-scale study by Mendis, Lehmann, and Gardner (2017), it was found that those adults with a care history who achieved a degree in higher education had been exposed to carers or significant people in their earlier lives who valued learning and education, and imparted the importance of these to them. Perhaps lots of talking about all manner of things in an engaged fashion is the key to developing the capacity to use language and narrative.

There has been considerable attention given to the issue of 'professional fostering' (McHugh \& Pell, 2013) in Australia and internationally, with a number of specific programmes designed to provide a higher level of therapeutic care through professional fostering arrangements. These are often associated with the care of children with challenging behaviours and therapeutic needs. However, there continue to be a number of practical approaches that are important in both general foster care and specialist caring. One is the development of the life story book, which was first talked about in the 1980s and is now a more formalised process promoted by government and non-government welfare organisations in most westernised countries (see for instance, http://www.community.nsw.gov.au/parents,-carers-andfamilies/fostering,-guardianship-and-adoption/fostercare/are-you-a-community-services-foster-carer/life-storywork, https://www.childprotection.sa.gov.au/childrencare/support-children-care/life-story-work and http:// familycaresociety.co.uk/services/life-story-project/). Much of this work with children and young people is done by their carers, many of whom may need training and encouragement to pursue not just the development of the life stories of those in their care, but the day-to-day conversations that encourage discussion of emotions, ideas and issues. It is finding language to describe the events of the day, the interactions with others, the challenges and the joys experienced that not only develop language, but assist with identification of feelings, enrich the understanding of them and provide narrative structure through which to make meaning of them.

As well as the day-to-day engagement in conversation, there is the exposure of children and young people to a range of experiences, and the importance of exposure to diverse cultural activities and events, and seeing the ways other people live. This does not mean expensive excursions and holidays, but, rather, being connected to community events like festivals, concerts and exhibitions and being encouraged to engage with surroundings - people, architecture, history and the natural environment. Exposure to literature in the home is also important, along with shared reading and discussions about stories and television programmes that promote children's reflections on differing perspectives, choices and possibilities. All of these approaches serve to enrich the language and narratives of children and young people and provide opportunities to pursue interests and talents as they grow older.

In developing this first Issue for 2018, a number of papers and topics were selected. We begin with a Commentary on a research study undertaken by Rachel Breman, Ann MacRae and Dave Vicary for Baptcare titled: 'It's been an absolute nightmare' - Family Violence in Kinship Care in Victoria'. Kinship care is the fastest growing form of OOHC care in many places and the preferred placement option for children who are unable to live with their parents. However, little is known about family violence in kinship care that is perpetrated by a close family member of the child in care (usually the child's mother/father) against the carer(s) and children once the placement has started. In 2017, Baptcare undertook research in Victoria with 101 kinship carers to gain a better understanding of how family violence was impacting on children and families. The study demonstrated that significant levels of violence from family members are being experienced by kinship carers and the children in their care. As a response, Baptcare is proactively addressing family violence in kinship care across a range of domains in order to provide solutions to the issues identified in this research.

This is followed by a second Commentary paper by Pamela Schulz, a longstanding colleague of the late Prof. Freda Briggs, which addresses the discourse of child protection and its ensuing political responses. The paper presents an analysis of media platforms, which have addressed the need for reform and associated recommendations for change, following child abuse and neglect reports. Clear evidence emerges of a disturbing discourse of deferral and political inaction that is only relieved when Inquiries and Royal Commissions advocate strong and immediate changes. In addition, this study considers the silences - the 'lacunae' or what is missing in text or discourse - to show that the voices of children tend only to be heard after the event of horrific abuse, and often only when whistle-blowers or advocates have come forward.

The first of our articles is by India Bryce, who addresses the issue of cumulative harm and examines a range of trends and research in order to identify current gaps in the legislative response to cumulative harm identification and intervention. From her analysis of the current policies informing child protection in Australia, it is clear that there is a shift towards a more holistic understanding of harm and the impacts of long-term maltreatment. However, Bryce concludes that a nationwide collaborative level of consistent practice, which places cumulative harm and reoccurring maltreatment on an equal footing with episodic maltreatment, particularly in relation to notification and reporting, has not yet been achieved.

Continuing the discussion of concern about harm to children is the article by Angelika Poulsen on intimate partner violence (IPV) which has been widely acknowledged 
as a prominent problem throughout Australia. A growing body of research has linked corporal punishment of children in the home with numerous adverse outcomes both in childhood and adulthood. Some of these adverse outcomes in childhood, such as aggression and antisocial behaviour, may be antecedents for involvement in violence as an adult. Poulsen discusses corporal punishment as a type of family violence that is legal in Australia; yet its role in the family violence scenario is not yet fully appreciated. This article presents the extant scientific literature on the link between corporal punishment in childhood and involvement in IPV in adulthood, and argues for the employment of this knowledge in the implementation of policies regarding corporal punishment of children.

Frank Ainsworth and Patricia Hansen follow the Bryce paper with an article that raises questions about the utility of renaming OOHC as Intensive Therapeutic Care (ITC). They question whether group homes can be therapeutic given the characteristics of the population of children and young people they accommodate, their small size, the staffing complement and the limited job satisfaction with high staff turnover as a consequence of this smallness. All of these factors are understood to lead to the well documented, anti-therapeutic instability of the group home life space, discussed in this paper.

Amy Gill and Grace Oakley, in their article titled 'Agency workers' perceptions of cross-system collaboration to support students in out-of-home care', focus on the ongoing issue of collaboration between government departments for the educational benefit of children and young people in care. This has been an area of concern for a long time and this study, based in Western Australia, found that education and child protection systems are able to minimise collaboration barriers through the development and implementation of mutually developed and agreed upon processes to support the unique educational needs of students in OOHC. However, challenges occurred due to financial constraints, difficulties obtaining mandated Documented Education Plans, and insufficient awareness among some educators of the child protection system and the impact of trauma on learning and behaviour.

Turning to the issue of research, an article titled 'Challenges and opportunities for innovation in child abuse and neglect research within the child welfare system in Australia', by Mohajer Hameed, provides a brief overview of the key methodological limitations, barriers and challenges, as well as the strengths of the research methods used in studying child abuse and neglect. His paper also explores the potential gains from adopting a national translational research framework. However, the innovative translation of research and knowledge into effective care system responses and evidence-based practices for children remains a pressing issue. If we are to make further advances in Australian research, the evidence base will require substantial investment in research and evaluation activities, with a new emphasis on translational research and active collabora- tion between researchers and practitioners. The paper concludes with key recommendations and directions for future Australian-based research, with the ultimate goal of improving practices and policies.

This is followed by two papers that reflect research investigations. First, Debbie Plath reports on a specialist multilevel programme called Getting On Track In Time! (Got It!) and the trajectories of a cohort of children and families for two years after they participated in the programme. Early behavioural problems which may lead to conduct disorder are of concern to parents and in educational settings, and can lead to anti-social and criminal behaviours if not addressed. The findings from this small study suggest that, for some children and families, the targeted group intervention programme was sufficient to address behaviour concerns. Other families require ongoing services in response to specialised needs. It appears that a successful early intervention programme for emerging conduct problems requires a targeted parenting intervention programme, strategies that facilitate access to specialised services, and attention to building capacities for the ongoing support of families within schools.

Secondly, Catherine Wade and her colleagues report on a project titled Parenting Today in Victoria (PTIV) which involved a survey carried out in 2015-2016. The survey was designed to explore the contemporary parenting experiences and service use of a large and representative sample of parents, with the aim of building understanding of parenting attitudes, behaviours and practices, concerns, and help-seeking, as well as to develop to a survey that could be repeated at intervals to measure and understand contemporary parenting experiences across Victoria over time. The project findings are intended to be used by decision-makers to inform policy and improve the service system in Victoria.

Finally, a book review by Frank Ainsworth provides comment on the publication titled Challenging the politics of early education. Who's 'saving' children and why written by Val Gillies, Rosalind Edwards, and Nicola Horsley.

\section{References}

Berzenski, S., \& Yates, T. (2017). The differential influences of parenting and child narrative coherence on the development of emotion recognition. Developmental Psychology, 53(10), 1912-1923. doi: 10.1037/dev0000395.

Gill, R. (2017). The extinction of creativity. Artlife, 26, 25.

Kelly, K., \& Bailey, A. (2012). Becoming independent storytellers: Modeling children's development of narrative macrostructure. First Language, 33(1), 68-88. doi: $10.1177 / 0142723711433582$.

Lum, J., Powell, M., \& Snow, P. (2018). The influence of maltreatment history and out-of-home-care on children's language and social skills. Child Abuse \& Neglect, 76, 65-74.

McCarthy, A. (2015). Telling stories, washing hands: Exploring the role of narrative in development programmes targeting children. South Asian History and Culture, 6(3), 401-416. doi: 10.1080/19472498.2015.1030876. 
McHugh, M., \& Pell, A. (2013). Reforming the foster care system in Australia: A new model of support, education and payment for foster parents. Melbourne: Berry Street/University of New South Wales. Retrieved from http://www.aph. gov.au/DocumentStore.ashx?id=013247a0-e100-49fb9665-82c7ac8b1350\&subId=301927.

Mendis, K., Lehmann, J., \& Gardner, F. (2017). Promoting academic success of children in care. The British Journal of Social Work, bcx029, 1-18. doi: 10.1093/bjsw/bcx029.

Pinto, G., Tarchi, C., \& Bigozzi, L. (2016). Development in narrative competences from oral to written stories in five- to seven-year-old children. Early Childhood Research Quarterly, 36, 1-10. doi: 10.1016/j.ecresq.2015. 12.001.

Reese, E., Myftari, E., McAnally, H. M., Chen, Y., Neha, T., Wang, Q., ... Robertson, S.-J. (2017). Telling the tale and living well: Adolescent narrative identity, personality traits, and well-being across cultures. Child Development, 88, 612628. doi: 10.1111/cdev.12618.

Stagnitti, K., \& Lewis, F. (2015). Quality of pre-school children's pretend play and subsequent development of semantic organization and narrative retelling skills. International Journal of Speech-Language Pathology, 17(2), 148-158. doi: 10.3109/17549507.2014.941934.

Stagnitti, K., Bailey, A., Stevenson, E., Reynolds, E., \& Kidd, E. (2016). An investigation into the effect of play-based instruction on the development of play skills and oral language. Journal of Early Childhood Research, 14(4), 389-406. doi: 10.1177/1476718X15579741.

Valentino, K., Nuttall, A., Comas, M., McDonnell, C., Piper, B., Thomas, T., \& Fanuele, S. (2014). Mother-child reminiscing and autobiographical memory specificity among preschool-age children. Developmental Psychology, 50(4), 1197-1207. doi: 10.1037/a0034912. 